\title{
¿Es tiempo de vacunar a la mujer embarazada contra la coqueluche?
}

\author{
José Cofré
}

\section{Is it time to vaccine pregnant woman against pertussis?}

Pertussis may cause death in neonates and very young infants. In these ages pertussis vaccine has not been administered yet or infants haven't received enough doses of vaccine to protect them. Cocoon strategy have been implemented in several countries and has limited efficacy because of low coverage among households. In vaccinated pregnant women transplacental transfer of pertussis antibodies starts at 32-34 weeks of pregnancy. United Kingdom, The United States of America and afterward other countries in Europe and America have adopted this strategy. Recent British research about security and effectiveness of pertussis vaccination in pregnant women to reduce pertussis incidence and fatality cases in neonate and very young infants infant support this strategy despite the fact that controlled studies about the efficacy and security in mothers and neonates have not been published. Several studies still not published or in progress are trying to answer these two questions and to evaluate possible interference between transplacentaly transferred pertussis antibodies and infants immune response to routine vaccines. Recently WHO has recommended pertussis vaccination during pregnancy in countries where neonatal pertussis is a public health problem. Nowadays, Chilean experts discuss if pertussis vaccination of pregnant women must be adopted in our country.

Key words: Pertussis, whooping tough, mortality, vaccination, pregnancy.

Palabras clave: Coqueluche, tos convulsiva, tos ferina, letalidad, vacunación, embarazo.

\section{¿Por qué vacunar contra coqueluche en el embarazo?}

Hay distintos argumentos que hacen planteable esta estrategia. Veamos:

- La enfermedad alcanza su máxima gravedad en el primer trimestre de la vida. Las tasas de letalidad por coqueluche en Chile, al igual que en países desarrollados, son bajas; en el año 2011 fue de 0,09/100.000 hbts, en el 2012 de 0,07/100.000 hbts, en el 2013 de 0,02/100.000 hbts y en el 2014 de 0,03/100.000 hbts. Eso significó, no obstante, que en el año 2011 fallecieron en Chile 16 lactantes bajo seis meses de edad; en el 2012 fueron 13/1.240 (letalidad: 1,04\%) en este grupo etario, en el año 2013 hubo 3 fallecidos, en el 2014 se notificaron 7 muertes en este mismo grupo etario y en el 2015 (hasta septiembre inclusive) se han registrado 7 fallecidos, todos bajo 6 meses de edad ${ }^{1,2}$.

- Si bien se ha documentado el paso de anticuerpos vía placentaria al recién nacido, ello no es frecuente dependiendo de la presencia de anticuerpos en la madre $^{3}$. Es discutible la utilidad de los anticuerpos anti-pertussis en la protección contra la enfermedad pero, como veremos más adelante pero, de alguna manera, la vacunación materna ha demostrado ser una estrategia eficiente para proteger al neonato.
- La vacunación del niño alcanza su mayor eficacia al cabo de tres a cuatro dosis de vacuna ${ }^{4}$; comenzando la vacunación al mes de vida y luego a los 3 meses, aún así no habrá suficiente protección durante el primer semestre de vida.

- Una estrategia alternativa, recomendada por expertos internacionales y puesta en práctica en nuestro país, para regiones con alta morbilidad por coqueluche-la llamada estrategia de capullo-es de difícil implementación y logros de coberturas sobre todos los contactos del neonato y potenciales fuentes de contagio ${ }^{5}$. En Chile se aplicó esta estrategia durante los años 2012 y 2013 en aquellas regiones administrativas que presentaran las más altas incidencias y letalidad por coqueluche; fueron Bío-Bío, O’Higgins, Valparaíso y Región Metropolitana. Se protocolizó vacunar a la madre y dos contactos intra-familares, en torno al recién nacido. La cobertura alcanzada fue de $91 \%$ en las puérperas y de $60 \%$ en otros contactos. Pudo observarse, durante el año 2013, que no hubo fallecidos por coqueluche. Detrás de este aparente éxito, queda la duda si esta ausencia de fallecidos fue consecuencia de la estrategia implementada o simplemente una casualidad pues la cobertura según protocolo fue baja en los contactos y aún más, pudo haber existido más de los dos contactos que considerara el protocolo, los que no fueron vacu-
Hospital Luis Calvo Mackenna, Santiago, Chile.

Unidad de Infectología, Servicio de Pediatría.

Conflictos de interés: El Dr. Cofré ha sido invitado por Sanofi Pasteur, Pfizer y Novartis a congresos de la especialidad de infectología, a reuniones nacionales $\mathrm{e}$ internacionales sobre vacunación y a reuniones científicas sobre productos elaborados por estas casas farmacéuticas. Ha recibido honorarios como expositor o moderador en actividades académicas organizadas por estas tres casas farmacéuticas.

Recibido: 30 de octubre de 2015 Aceptado: 26 de noviembre de 2015

Correspondencia a: José Cofré Guerra pepecofre@gmail.com 
nados; si así fuera, las coberturas habrían sido todavía más bajas. Es opinión de este autor, que difícilmente una vacunación en capullo con una cobertura inferior a $60 \%$ pudiera considerarse responsable de esta caída tan acentuada en la letalidad. R. Libster y KM Edwards han comentado que la estrategia de capullo no es adecuada para un escenario en que las coberturas son deficientes ${ }^{6}$. Señalan, además, estos dos autores, que la vacunación en capullo entraña una latencia para inducir inmunidad en la madre y los contactos en torno al neonato, dejando una "ventana" de personas susceptibles por un plazo significativo y crítico (las dos o tres primeras semanas de vida del neonato) ${ }^{6}$.

- También se ha intentado evitar el contagio de los neonatos y lactantes que no están en edad de vacunar aún, mediante la vacunación de escolares y adolescentes, con el sustento teórico que la inmunidad otorgada por las vacunas se extingue al cabo de 4-6 años y, en consecuencia, los escolares y adultos jóvenes se tornarían susceptibles actuando como una fuente de contagio intrafamiliar para sus hermanos menores ${ }^{7}$. Esta estrategia ha sido propuesta ${ }^{8}$ y luego recomendada internacionalmente ${ }^{9}$ y adoptada por nuestro país, entre otros. Desde el año 2011 y el 2103 se introdujo la vacunación de escolares en el $2^{\circ}$ año básico de estudio y en $8^{\circ}$ año básico, sucesivamente, lográndose coberturas crecientes de $70-88 \%$ para el $1^{\circ}$ básico y $80-85 \%$ para el $8^{\circ}$ año básico. (Comunicación personal. Cecilia González, PNI-MINSAL). Esta estrategia ha sido evaluada como satisfactoria para reducir la enfermedad entre las cohortes vacunadas, sin impactar mayormente en los neonatos y lactantes pequeños, el foco principal de las medidas epidemiológicas para el control de la coqueluche $^{10}$.

Analicemos, a continuación, los fundamentos positivos para considerar la vacunación de la mujer durante la gestación.

- La vacunación de la mujer gestante induce anticuerpos contra los componentes de la vacuna anti-pertussis (pertusinógeno, hemaglutinina filamentosa, pertactina, aglutinógenos), que atraviesan la placenta, a partir de las 32-34 semanas de gestación ${ }^{11,12}$.

\section{¿Hay experiencias históricas con esta estrategia?}

- Este pensamiento estuvo presente desde los albores del desarrollo de la vacuna con células enteras. Hubo múltiples ensayos ${ }^{13}$ que luego se abandonaron por las reacciones locales observadas y el temor de efectos nocivos sobre el hijo en gestación.

\section{¿Vacunas celulares o acelulares debieran usarse?}

Siempre acelulares -Tdpa (vacuna con baja carga antigénica de toxoide diftérico y de componentes anti- pertussis, apropiada para usar sobre 7 años de edad)- pues no es recomendable usar vacuna celular más allá de los siete años de edad, existe un riesgo incrementado de efectos adversos sobre esa edad.

\section{¿Porqué no vacunar a la mujer en edad fértil temprana, antes de embarazarse?}

La respuesta inmune a la vacuna acelular produce una protección que es transitoria, como lo demostró, durante un brote epidémico, la caída en la eficacia de la vacuna en escolares, desde $75 \%$ inicial a $34,5 \%$ al cabo de dos años post vacunación ${ }^{14}$. La vacunación anticipada en una mujer en edad fértil, con el potencial de embarazarse en el futuro, no asegura que los anticuerpos anti-pertussis tengan aún una concentración suficiente para evitar la enfermedad de su hijo ${ }^{11,12,15}$. De manera que la vacuna debe administrarse a partir de las 28 semanas de gestación, con la intención de otorgar inmunidad al hijo que nacerá, ya desde las 32-34 semanas de embarazo ${ }^{16}$. Antes de ese tiempo de gestación, no hay paso de IgG materna a través de la placenta.

\section{¿Qué seguridad ofrece la administración de vacunas durante la gestación?}

La seguridad de esta vacuna ha sido evaluada por los ingleses comprobando en un estudio doble ciego y randomizado, preliminar, que no hubo mayores eventos adversos en 33 mujeres que recibieron la vacuna ni en sus hijos, en comparación con un grupo control no vacunado ${ }^{17}$. Luego, tras la aplicación de la vacuna en 20.074 mujeres embarazadas, no se observaron eventos adversos mayores en la madre ni en su hijo ${ }^{18}$.

\section{¿Qué eficacia se espera obtener vacunando mujeres en su gestación?}

Se espera, en la medida que la vacunación de las madres gestantes alcance una alta cobertura, que se minimicen las muertes por coqueluche en neonatos y lactantes bajo 3 meses y no se produzcan muertes por esta causa en hijos nacidos de madres vacunadas durante la gestación.

Hasta ahora se dispone de un estudio observacional llevado a cabo en Inglaterra, el que constató una eficacia de $91 \%$ en reducir la mortalidad por coqueluche en lactantes bajo 3 meses y de $90 \%$ si se consideran sólo lactantes bajo 2 meses de edad ${ }^{19}$. Recientemente, se publicó un estudio caso-control efectuado en Inglaterra y Gales, que confirma estas cifras ${ }^{20}$. Actualmente hay bajo el alero del National Institute of Health de los Estados Unidos de América, siete nuevos estudios en desarrollo, cuatro proyectos por iniciarse y dos estudios finalizados, que buscan documentar la eficacia y seguridad de esta estrategia, en la madre $\mathrm{y}$ en su hijo en gestación ${ }^{21-24}$. 


\section{¿Qué países han adoptado esta estrategia de vacunación?}

Inicialmente fue Gran Bretaña quien adoptó en el año 2012 esta estrategia, aún sin estar licenciada la vacuna para uso durante el embarazo ${ }^{25}$. De igual manera han procedido Estados Unidos de Norteamérica ${ }^{26}$, otras naciones del Commonwealth of Nations: Canadá, Irlanda, Nueva Zelandia, Australia, varios otros países europeos ${ }^{27}$, Israel $^{28}$ y en Latinoamérica: Argentina ${ }^{29,30}$, Costa Rica ${ }^{31}$, México $^{32}$, Uruguay ${ }^{33}$, Colombia ${ }^{34}$, entre otras, sobrepasando los registros sanitarios de sus respectivos países, en consideración al dramático problema de salud pública que representa la coqueluche en neonatos y lactantes y a las escasas perspectivas de controlar la enfermedad aplicando otras maneras de calendarizar la vacunación, llámese programas en el escolares, adolescentes y adultos.

\section{¿Qué dice la Organización Mundial de la Salud al respecto?}

En una publicación reciente (28 de agosto de 2015) dice textual:

"Vaccination of pregnant woman is likely to be the most cost-effective additional strategy for preventing disease in infants too young to be vaccinated and appears to be more effective and favourable than cocooning. National programmes may consider the vaccination of pregnant women with 1 dose of Tdap (in the $2^{\text {nd }}$ or 3rd trimester and preferably at least before the end of pregnancy) as a strategy additional to routine primary infant pertussis vaccination in countries or setting with high or increasing infant morbidity/mortality from pertussis ${ }^{\prime 35}$.

\section{Con todos estos antecedentes, ¿qué falta conocer para hacer de esta estrategia una recomendación universal?}

Dos aspectos suscitan preocupación aún: ¿será necesario revacunar a la mujer gestante nuevamente en cada embarazo? La presencia de anticuerpos anti-pertussis en las primeras semanas de vida iinterfiere con la respuesta inmune a las vacunas a colocar a partir de los meses de edad?

- La información disponible permite recomendar que, conocida la rápida caída del título de anticuerpos anti- pertusinógeno en la persona vacunada con $\mathrm{Tdpa}^{11,12,15}$, se ofrezca revacunación durante cada nuevo embarazo; no se conoce la seguridad de este proceder aunque la escasa información disponible orienta hacia su inocuidad $^{13}$.

- Evidencia preliminar existe sobre la inhibición de la respuesta inmune a las vacunas del lactante si ella se practica con vacunas celulares, no así si la vacuna empleada contiene pertussis acelular ${ }^{36}$. Esta observación debe ser confirmada o descartada con los mencionados estudios en marcha ${ }^{21-24}$.

\section{Resumen}

La coqueluche puede ser letal en neonatos y lactantes en sus primeros meses de vida. En estas edades, la vacunación anti Bordetella pertussis no ha sido administrada aún o completada hasta lograr la protección del niño. La estrategia de capullo implementada en diversas naciones es de limitada eficacia por la dificultad que entraña alcanzar altas coberturas entre los contactos de los neonatos. Surge como una posibilidad la vacunación en la mujer embarazada, estrategia fundamentada en el efectivo paso de anticuerpos maternos al neonato a partir de las 32-34 semanas de gestación. El Reino Unido, los Estados Unidos de América y luego otros países en Europa y América, han adoptado esta estrategia de vacunación. Publicaciones recientes provenientes del Reino Unido, sobre la seguridad y efectividad de la vacunación en la mujer embarazada, para evitar casos fatales de coqueluche, apoyan estas decisiones adoptadas pese a no disponerse de estudios controlados en el binomio madre-hijo. Múltiples estudios finalizados pero no publicados o aún en desarrollo, pretenden responder estas dos preguntas y evaluar además si los anticuerpos que atraviesan la placenta interfieren sobre las vacunas que serán administradas al lactante en los primeros meses de vida. Recientemente, la Organización Mundial de la Salud ha recomendado considerar la vacunación durante el embarazo en países donde la coqueluche es un importante problema de salud pública en neonatos. Se discute en Chile si es la vacunación anti-pertussis en la mujer gestante una estrategia a adoptar.

\section{Referencias bibliográficas}

1.- González C. Coqueluche: situación en Chile. Vacunas completas y acelulares. Presentación en: XXII Curso Internacional de Infectología y Terapia Antimicrobiana del Sur y II Symposium Internacional de Vacunas. Temuco, 4-6 de junio, 2015.

2.- Departamento de Epidemiología, MINSAL. Boletín Epidemiológico Trimestral. Enero a septiembre de 2015. 2015; 111, $\mathrm{N}^{\circ} 4$

3.- Van Rie A, Wendelboe A M, Englund J A. Role of maternal pertussis antibodies in infants. Pediatr Infect Dis J 2005; 24 (5): S62-S5.

4.- Edwards K M, Decker M D. Pertussis vaccines. E. Vaccines Plotkin SA, Orenstein WA, Offit PA. Eds. 2013. 6th ed. Elsevier-Saunders. pp: 447-92.

5.- Castagnini L A, Healy M, Rench M A, Wootton S H, Muñoz F M, Baker C J. Impact of maternal postpartum tetanus diphtheria toxoids and acellular pertussis immunization on infant pertussis infection. Clin Infect Dis 2012; 54 (1 Jan): 78-84.

6.- Libster R, Edwards K M. How can we best prevent pertussis in infants? Editorial Commentary. Clin Infect Dis 2012; 54 (1): 85-7.

7.- Cofré J. Vacunas anti-pertussis: acelular versus celular. ¿Acaso un regreso al pasado? Rev Chilena Infectol 2015; 32 (5): 559-63. 
8.- Van Rie A, Hethcote H W. Adolescent and adult pertussis vaccination: computer simulations of five new strategies. Vaccine 2004; 22 (23-24): 3154-65.

9.- Guiso N, Liese J, Plotkin S. The Global Pertussis Initiative. Meeting Report From the Fourth Regional Roundtable Meeting, France. April 14-15 2010. Human Vaccines 2011; 7 (4): 481-8.

10.- Quinn H E, McIntyre P B. The impact of adolescent pertussis immunization, 2004-2009: lessons from Australia. Bull World Health Organ 2011; 89: 666-74 | doi:10.2471/BLT.11.086538.

11.- Healy C M, Muñoz F M, Rench M A, Halasa N B, Edwards K M, Baker C J. Prevalence of pertussis antibodies in maternal delivery, cord, and infant serum. J Infect Dis 2004; 190 (15 July): $335-40$.

12.- Healy C M, Rench M A, Baker C J. Importance of timing of maternal combined tetanus, diphtheria and acellular pertussis (Tdap) immunization and ptrotection of young infants. Clin Infect Dis 2013; (February 15): 56: $539-44$.

13.- Mooi F R, de Greff S C. The case of maternal vaccination against pertussis. Lancet Infect Dis 2007; 7: 614-24.

14.- Weston W. Messier M, Friedland L R, Wu X, Howe B. Persistence of antibodies 3 years after booster vaccination of adults with combined acellular pertussis, diphtheria and tetanus toxoid vaccine. Vaccine 2011; 29; 8483-6.

15.- Koepke R, Eickhoff J C, Ayele R A, Petit A B, Schauer S L, Hopfensperger D J, et al. Estimating the effectiveness of diphtheria tetanus acellular pertussis vaccine (Tdap) for preventing pertussis: evidence of rapidly waning immunity and difference in effectiveness by Tdap brand. J Infect Dis 2014; 10: 942-53.

16.- CDC. Updated recommendations for use of tetanus toxoid, reduced diphtheria toxoid and acellualar pertussis vaccine (Tdap) in pregnant women. Advisory Committee on Immunization Practices (ACIP) 2012. MMWR Morbid Mortal Wkly Rep Recommendations and Reports. 2013; 62 (February 22; (7); 131-5.

17.- Muñoz F M, Bond N H, Maccato M, Pinell P, Hammill H A, Swamy G K, et al. Preliminary Communication. Safety and immunogenicity of tetanus diphtheria an acellular pertussis (Tdap) immunization during pregnancy in mothers and infants. A randomized clinical trial. JAMA 2014; 311 (17): 1760-9.

18.- Donegan K. Safety of pertussis vaccination in pregnant women in UK: observational study. $\mathrm{Br}$ Med J 2014; 349:g4219doi:10.1136/bmj.g4219.
19.- Amirthalingam G, Andrews N, Campbell H, Ribeiro S, Kara E, Donegan K, et al. Effectiveness of pertussis maternal vaccination in England: an observational study. Lancet 2014; 384 (October): 1521-8.

20.- Dabrera G, Amirthalingam G, Andrews N, Campbell H, Ribeiro S, Kara E, et al. A case-control study to estimate the effectiveness of maternal pertussis vaccination in protecting newborn infants in England and Wales, 2012-2013. Clin Infect Dis 2015; 60 (3): 3333-7.

21.- https://clinicaltrials.gov/ct2/results?term=pertus sis + vaccine + AND + pregnancy \&pg $=1$

22.- National Institute of Allergy and Infectious Diseases. (NIAID). Safety and immunogenicity of Tdap vaccine in healthy pregnant women, safety in their neonates and effect of maternal immunization on infant immune response to DTaP vaccine. Disponible en: http:// clinicaltrials.gov/show/NCT00707148?term= pertussis + vaccine + AND + pregnancy \& rank $=9$ (Accedido el 26 de octubre de 2015).

23.- Halperin S. Pertussis maternal immunization study. Disponible en: http://clinicaltrials.gov/ show/

24.- Ramírez-Aranda J M, et al. Immunogenicity and safety of an acellular DPT vaccine during pregnancy. Disponible en: https://clinicaltrials. gov/ct2/show/NCT01445743?term=pertussis ${ }^{+}$ vaccine + AND + pregnancy\&rank $=14$ (Accedido el 26 de octubre de 2015).

25.- Pertussis Vaccination Programme for Pregnant Women: vaccine coverage estimates in England, September to December 2014. Health Protection Report. Weekly Report 2015; 9; 7 (27 February): 7-27.

26.- CDC. Updated recommendations for use of tetanus toxoid, reduced diphtheria toxoid and acellular pertussis vaccine (Tdap) in pregnant women in persons who have or anticipate having close contact with an infant aged $<12$ months. Advisory Committee on Immunization Practices (ACIP) 2011. MMWR 2011; 60: 1424-6.

27.- European Centre for Disease Prevention and Control. Vaccine schedule. Recommended immunization for pertussis. http://vaccineschedule.ecdc.europa.eu/Pages/Scheduler.aspx (Accedido el 29 de octubre de 2015).

28.- State of Israel. Ministry of Health. Vaccines for women before pregnancy, during pregnancy and after childbirth. http://www.health.gov. il/English/Topics/Pregnancy/during/Pages/ vaccine_pregnant.aspx (Accedido el $29 \mathrm{de}$ octubre de 2015).
29.- Calendario Nacional de Vacunación 2015. http://www.msal.gob.ar/index.php/component/ content/article/46-ministerio/184-calendarionacional-de-vacunacion-2014. (Accedido el 29 de octubre de 2015).

30.- Vizzotti C, Neyro S, Katz N, Juárez M V, Perez Carrega M E, Aquino A, et al. Maternal immunization in Argentina: A storyline from the prospective of a middle income country. Vaccine (2015), http://dx.doi.org/10.1016/j. vaccine.2015.07.109.

31.- Ministerio de Salud de Costa Rica. Norma Nacional de Vacunación 2013-2014. http://www.ministeriodesalud.go.cr/ index.php/vigilancia-de-la-salud/normasprotocolos-y-guias/2302-norma-nacionalde-vacunacion-2013/file (Accedido el 29 de octubre de 2015).

32.- Estados Unidos Mexicanos. Gobierno Federal. Guía de Práctica Clínica. Vacunación en la embarazada. http://www.cenetec.salud.gob. $\mathrm{mx} /$ descargas/gpc/CatalogoMaestro/580 GPC Vacunacixnenlaembarazada/580GER.pdf (Accedido el 29 de octubre de 2015).

33.- Ministerio de Salud Pública. República Oriental del Uruguay. Comunicado a personal de salud. Vacunación con dpaT a embarazadas. http:// www.msp.gub.uy/noticia/comunicado-personalde-salud-vacunaci $\% \mathrm{C} 3 \% \mathrm{~B} 3 n$-con-dpatembarazadas (Accedido el 29 de octubre de 2015)

34.- República de Colombia. Ministerio de Salud y Protección Social. Lineamiento Estratégico para la Introducción de la Vacuna TdaP (TétanosDifteria-Tos ferina acelular) en el Esquema del Programa Ampliado de Inmunizaciones-PAI Para Mujeres Gestantes de las cohortes 2013 y 2014. Colombia 2013. https://www.minsalud. gov.co/Documentos\%20y\%20Publicaciones/ lineamiento $\% 20$ Estrat $\% \mathrm{C} 3 \%$ A9gico $\% 20$ para $\% 201 \mathrm{a} \% 20$ Introducci $\% \mathrm{C} 3 \% \mathrm{~B} 3 \mathrm{n} \% 20 \mathrm{de} \% 20$ 1a\%20Vacuna\%20TdaP.pdf (Accedido el 29 de octubre de 2015)

35.- World Health Organization. Pertussis vaccines: WHO position paper-August 2015. Weekly Epidemiological Record. 2015 (N: 35); 90: 433-60. http://www.who.int/wer/2015/wer9035. pdf?ua $=1$

36.- Englund J A, Anderson E L, Reed G F, Decker M D, Edwards K M, Pichichero M E, et al. The effect of maternal antibody on the serologic response and the incidence of adverse reactions after primary immunization with acellular and whole-cell pertussis vaccines combined with diphtheria and tetanus toxoids. Pediatrics 1995; 96 (3): S580-S4. 